\title{
Global Analysis of an SEIR Epidemic Model with a Ratio-Dependent Nonlinear Incidence Rate
}

\author{
Xiaomei Ren, Tiansi Zhang \\ College of Science, University of Shanghai for Science and Technology, Shanghai, China \\ Email: zhangts1209@163.com
}

How to cite this paper: Ren, X.M. and Zhang, T.S. (2017) Global Analysis of an SEIR Epidemic Model with a Ratio-Dependent Nonlinear Incidence Rate. Journal of Applied Mathematics and Physics, 5, 2311-2319. https://doi.org/10.4236/jamp.2017.512188

Received: October 10, 2017

Accepted: December 4, 2017

Published: December 7, 2017

Copyright $\odot 2017$ by authors and Scientific Research Publishing Inc. This work is licensed under the Creative Commons Attribution International License (CC BY 4.0).

http://creativecommons.org/licenses/by/4.0/

\begin{abstract}
In this paper, a SEIR model with ratio-dependent transmission rate in the form $\frac{k S^{h} I}{S^{h}+\alpha I^{h}}$ is studied and the basic reproduction number which determines the disease's extinction or continued existence is obtained. By constructing the proper Lyapunov function, we prove that if $R_{0} \leq 1$, the disease-free equilibrium point of the model is globally asymptotically stable and the disease always dies out; if $R_{0}>1$, the endemic equilibrium point is globally asymptotically stable and the disease persists.
\end{abstract}

\section{Keywords}

SEIR Model, the Ratio-Dependent Transmission Rate, Basic Reproduction Number, Equilibrium, Global Stability

\section{Introduction}

As we all know, infectious diseases are enemies of human health. For a long time, people have been fighting various infectious disease; and many methods have been used to study the spread of infectious diseases, so as to control and eliminate infectious diseases, see [1]. Amongst them, mathematical model has become an important tool to analyze the epidemiological characteristics of infectious diseases since the pioneer work of Kermack and McKendrick [2] [3], which provides us useful control measures in [4]. In standard epidemiological models, the incidence rate (the rate of new infections) is bilinear in the infective and susceptible individuals, see [5] [6] [7]. It has been suggested that the diseases transmission process may have a nonlinear incidence rate, see [8] [9] [10].

In real life, epidemics tend to have an incubation period, as susceptible to infection after contacting with infected people. First of all, carrying virus, the virus 
is not immediately, but after a period of time, to onset and into the herd of infected people. In the paper, on the basis of the work of Yuan and Li in [11] an SEIR (Susceptible-Exposed-Infected-Removed) epidemic model is considered with a ratio-dependent nonlinear incident rate $g(S, I)$.

The transmission function $g(S, I)$ plays a key role in determining disease dynamics, see [12] [13]. Traditionally, the density-dependent transmission (or the bilinear incidence rate, $g(S, I)=k S I, k$ the proportionality constant) and the frequency-dependent transmission (or the standard incidence rate, $\left.g(S, I)=\frac{k S I}{S+I}\right)$ are two extreme forms of disease transmission, which have been frequently used in well-know epidemic models [14] [15]. For example, Capasso and Serio [16] introduced a saturated transmission rate $g(S, I)=f(I) S$, where the infectious force $f(I)$ is a function of infectious individuals which has been used in many classic disease model. Especially, Yuan and Li [11] studied a rate-dependent nonlinear incident rate with the following form

$$
g(S, I)=f\left(\frac{I}{S}\right) S=\frac{k\left(\frac{I}{S}\right)^{l} S}{1+\alpha\left(\frac{I}{S}\right)^{h}}=\frac{k S^{h-l+1} I^{l}}{S^{h}+\alpha I^{h}},
$$

where $\alpha$ is a parameter which measures the psychological or inhibitory effect. It should be noted that if $\alpha=1$ and $h=l=1$, (1) becomes the well-known frequency-dependent transmission rate $\frac{k S I}{S+I}$.

In the case of $l=1$, we can obtain the rate-dependent transmission rate $g(S, I)$ as the following form:

$$
g(S, I)=f\left(\frac{I}{S}\right) S=\frac{k I}{1+\alpha\left(\frac{I}{S}\right)^{h}}=\frac{k S^{h} I}{S^{h}+\alpha I^{h}},
$$

which indicates that the transmission rate of disease is approximately governed by $k I$ if $\frac{I}{S}$ is small (e.g., at the beginning of disease's spreading) or it is approximately governed by $\frac{k}{\alpha}\left(\frac{S}{I}\right)^{h-1} S$ if $\frac{I}{S}$ is large (e.g., in the endemic when almost everyone is infected). Therefore, the ratio-dependent transmission rate (2) indeed takes accounts of the crowding effects and behavior changes during epidemics. In this paper, we mainly focus on a SEIR epidemic model with the ratio-dependent incidence rate (2).

We consider the global properties of this SEIR model and show that if the basic reproduction number $R_{0} \leq 1$, the disease-free equilibrium point is globally asymptotically stable, while if $R_{0}>1$, the disease-free equilibrium point is unstable and the unique endemic equilibrium point is globally asymptotically stable.

The organization of this paper is as follows: in the next section, we present the 
model and derive the disease-free equilibrium point and the endemic equilibrium point. In Section 3 we analyze the global stability of the equilibrium point. A brief discussion and summarize are given in Section 4.

\section{Model Formulation}

The whole population $N(t)$ is divided into four subclasses based on disease status: the susceptible population, the exposed population, the infected population and removed population, denoted by $S(t), E(t), I(t), R(t)$, respectively, and $N(t)=S(t)+E(t)+I(t)+R(t)$. We assume that infectious disease can cause additional mortality, then the SEIR model can be modeled by the following set of nonlinear differential Equations (3) deterministically:

$$
\left\{\begin{array}{l}
\frac{\mathrm{d} S}{\mathrm{~d} t}=A-\frac{k S^{h} I}{S^{h}+\alpha I^{h}}-\mu S, \\
\frac{\mathrm{d} E}{\mathrm{~d} t}=\frac{k S^{h} I}{S^{h}+\alpha I^{h}}-(\mu+q) E, \\
\frac{\mathrm{d} I}{\mathrm{~d} t}=q E-(\mu+\delta+\gamma) I, \\
\frac{\mathrm{d} R}{\mathrm{~d} t}=\gamma I-\mu R,
\end{array}\right.
$$

where $A$ is the recruitment rate of the population, $\mu$ is the natural death rate, $q$ is the constant rate such that the exposed individuals become infective, $\gamma$ is the constant rate for recovery, $\delta$ is the disease inducing death. Since $R$ does not appear in the first three equations of system (3), thus (3) reduces to the following three-dimensional system (4)

$$
\left\{\begin{array}{l}
\frac{\mathrm{d} S}{\mathrm{~d} t}=A-\frac{k S^{h} I}{S^{h}+\alpha I^{h}}-\mu S, \\
\frac{\mathrm{d} E}{\mathrm{~d} t}=\frac{k S^{h} I}{S^{h}+\alpha I^{h}}-(\mu+q) E, \\
\frac{\mathrm{d} I}{\mathrm{~d} t}=q E-(\mu+\delta+\gamma) I .
\end{array}\right.
$$

Because $\left.\frac{\mathrm{d} S}{\mathrm{~d} t}\right|_{S=0}=A>0,\left.\frac{\mathrm{d} E}{\mathrm{~d} t}\right|_{E=0}=\frac{k S^{h} I}{S^{h}+\alpha I^{h}} \geq 0,\left.\frac{\mathrm{d} I}{\mathrm{~d} t}\right|_{I=0}=q E \geq 0$, the non-negativity of the initial value of system (4) in $R_{+}^{3}$ is guaranteed, where

$$
R_{+}^{3}=\{(S, E, I): S>0, E>0, I>0\},
$$

It follows from system (4) that:

$$
\begin{aligned}
\frac{\mathrm{d}(S+E+I)}{\mathrm{d} t} & =A-\mu S-(\mu+q) E+q E-(\mu+\delta+\gamma) I \\
& =A-\mu S-\mu E-(\mu+\delta+\gamma) I \\
& =A-\mu(S+E+I)-(\delta+\gamma) I \\
& \leq A-\mu(S+E+I) .
\end{aligned}
$$

Since $\lim _{t \rightarrow+\infty} \sup (S+E+I) \leq \frac{A}{\mu}$, the feasible region for system (4) is thus a 
bounded set $\Gamma$ :

$$
\Gamma=\left\{(S, E, I): S+E+I \leq \frac{A}{\mu}, S>0, E \geq 0, I \geq 0\right\} .
$$

The region $\Gamma$ is positively invariant with respect to systems (4). So, the only solution with the associated initial conditions will be considered inside the region $\Gamma$, where the uniqueness of solutions, usual existence, and continuation results are satisfied. Hence, system (4) is considered mathematically and epidemiologically well posed in $\Gamma$. Notice that model (4) has a disease-free equilibrium point $P_{0}=\left(\frac{A}{\mu}, 0,0\right)$ for all parameter values. Let $X=(E, I, S)^{\mathrm{T}}$, system (4) can be written as (see [17])

$$
\frac{\mathrm{d} X}{\mathrm{~d} t}=\mathcal{F}(X)-\mathcal{V}(X)
$$

And

$$
\begin{gathered}
\mathcal{F}(X)=\left(\begin{array}{c}
\frac{k S^{h} I}{S^{h}+\alpha I^{h}} \\
0 \\
0
\end{array}\right), \\
\mathcal{V}(X)=\left(\begin{array}{c}
(\mu+q) E \\
-q E+(\mu+\delta+\gamma) I \\
-A+\frac{k S^{h} I}{S^{h}+\alpha I^{h}}+\mu S
\end{array}\right) .
\end{gathered}
$$

The jacobian matrices of $\mathcal{F}(X)$ and $\mathcal{V}(X)$ at the disease-free equilibrium point $P_{0}$ are, respectively,

$$
\begin{aligned}
& D \mathcal{F}\left(P_{0}\right)=\left(\begin{array}{ll}
F & 0 \\
0 & 0
\end{array}\right), \\
& D \mathcal{V}\left(P_{0}\right)=\left(\begin{array}{ll}
V & 0 \\
J_{1} & J_{2}
\end{array}\right),
\end{aligned}
$$

where

$$
\begin{gathered}
F=\left(\begin{array}{ll}
0 & k \\
0 & 0
\end{array}\right), \\
V=\left(\begin{array}{cc}
\mu+q & 0 \\
-q & \mu+\delta+\gamma
\end{array}\right) .
\end{gathered}
$$

So the regeneration matrix of system (4) is

$$
Q=F V^{-1}=\left(\begin{array}{cc}
\frac{k q}{(\mu+q)(\mu+\delta+\gamma)} & \frac{k}{\mu+\delta+\gamma} \\
0 & 0
\end{array}\right),
$$

the spectral radius of $F V^{-1}$ is

$$
\rho\left(F V^{-1}\right)=\frac{k q}{(\mu+q)(\mu+\delta+\gamma)} .
$$


Hence the basic reproduction number $R_{0}$ (see [18]) is

$$
R_{0}=\frac{k q}{(\mu+q)(\mu+\delta+\gamma)} .
$$

Without difficulty we can get unique endemic equilibrium point state $P^{*}=\left(S^{*}, E^{*}, I^{*}\right)$ of model (4) with

$$
\left\{\begin{array}{l}
S^{*}=\frac{A}{\mu}-\frac{(\mu+q)(\mu+\delta+\gamma) I^{*}}{\mu q}, \\
E^{*}=\frac{(\mu+\delta+\gamma) I^{*}}{q}, \\
I^{*}=\left(\frac{k S^{* h} q}{\alpha(\mu+q)(\mu+\delta+\gamma)}-\frac{S^{* h}}{\alpha}\right)^{\frac{1}{h}},
\end{array}\right.
$$

and the endemic equilibrium point is written in the following form:

$$
\left\{\begin{array}{l}
S^{*}=\frac{A R_{0}}{R_{0} \mu+k\left(\frac{R_{0}-1}{\alpha}\right)^{\frac{1}{h}}}, \\
E^{*}=\frac{k A\left(\frac{R_{0}-1}{\alpha}\right)^{\frac{1}{h}}}{I^{*}=} \frac{(\mu+q)\left(R_{0} \mu+k\left(\frac{R_{0}-1}{\alpha}\right)^{\frac{1}{h}}\right]}{A R_{0}\left(\frac{R_{0}-1}{\alpha}\right)^{\frac{1}{h}}} \\
R_{0} \mu+k\left(\frac{R_{0}-1}{\alpha}\right)^{\frac{1}{h}}
\end{array}\right.
$$

if $R_{0}>1$.

Through the above analysis, system (4) has no endemic equilibrium point for $R_{0} \leq 1$, and from (19) we know that system (4) has a unique endemic equilibrium point if $R_{0}>1$.

\section{Global Stability of the Equilibrium Point}

In this section, we first consider the global stability of model (4) at the disease-free equilibrium point $P_{0}$.

Theorem 1: If $R_{0} \leq 1$, the disease-free equilibrium point $P_{0}$ is globally asymptotically stable; if $R_{0}>1, P_{0}$ is unstable.

Proof. The characteristic equation of system (4) at $P_{0}$ is give by

$$
(\lambda+\mu)\left[\lambda^{2}+(2 \mu+q+\delta+\gamma) \lambda+(\mu+q)(\mu+\delta+\gamma)-k q\right]=0 .
$$

It is clear that $\lambda=-\mu$ is one root of (20). The other roots of (20) are determined by the following equation 


$$
\lambda^{2}+(2 \mu+q+\delta+\gamma) \lambda+(\mu+q)(\mu+\delta+\gamma)-k q=0 .
$$

If $R_{0}<1,(\mu+q)(\mu+\delta+\gamma)-k q>0$, thus (21) has two roots with negative real parts, therefore, the disease-free equilibrium point $P_{0}$ is locally asymptotically stable.

To complete the proof, we construct the following Lyapunov function

$$
L_{1}=q E+(\mu+q) I .
$$

The time derivative of $L_{1}$ along the solution of (4) is

$$
\begin{aligned}
\frac{\mathrm{d} L_{1}}{\mathrm{~d} t} & =q \frac{\mathrm{d} E}{\mathrm{~d} t}+(\mu+q) \frac{\mathrm{d} I}{\mathrm{~d} t} \\
& =q \frac{k S^{h} I}{S^{h}+\alpha I^{h}}-(\mu+q) q E+(\mu+q) q E-(\mu+q)(\mu+\delta+\gamma) I \\
& =q \frac{k S^{h} I}{S^{h}+\alpha I^{h}}-(\mu+q)(\mu+\delta+\gamma) I \\
& \leq k q I-(\mu+q)(\mu+\delta+\gamma) I \\
& =[k q-(\mu+q)(\mu+\delta+\gamma)] I .
\end{aligned}
$$

When $R_{0} \leq 1$, we have $\frac{\mathrm{d} L_{1}}{\mathrm{~d} t} \leq 0$. By the LaSalle's invariance principle, see [19], we conclude that $P_{0}$ is globally asymptotically stable if $R_{0} \leq 1$.

When $R_{0}>1$, the Jacobian matrix of model (4) evaluated at $P_{0}$ is

$$
J\left(P_{0}\right)=\left(\begin{array}{ccc}
-\mu & 0 & -k \\
0 & -(\mu+q) & k \\
0 & q & -(\mu+\delta+\gamma)
\end{array}\right),
$$

which has an eigenvalue $-\mu<0$. Denoted by

$$
B=\left(\begin{array}{cc}
-(\mu+q) & k \\
q & -(\mu+\delta+\gamma)
\end{array}\right),
$$

we find that $\operatorname{tr} B<0$ ( $\operatorname{tr} B$ represents the trace of matrix $B$, which is the sum of the elements of the main diagonal of the matrix $B$ ). When $R_{0}>1$, and $\operatorname{det} B<0$ ( $\operatorname{det} B$ is the determinant of matrix $B$ ), the matrix $B$ must have a positive eigenvalue, thus the disease-free equilibrium point $P_{0}$ is unstable whenever $R_{0}>1$. This completes the proof.

For the stability of endemic equilibrium point $P^{*}=\left(S^{*}, E^{*}, I^{*}\right)$ of model (4), we have the following theorem:

Theorem 2: If $R_{0}>1$, the unique endemic equilibrium point $P^{*}=\left(S^{*}, E^{*}, I^{*}\right)$ of model (4) is globally asymptotically stable.

Proof. Suppose that $(S, E, I)$ is any positive solution to system (4). Define a Lyapunov function

$$
L_{2}(S, E, I)=\left|S-S^{*}\right|+\left|E-E^{*}\right|+\left|I-I^{*}\right| .
$$

Obviously $L_{2}\left(P^{*}\right)=0$ and when $P \neq P^{*}, L_{2}(P) \neq 0$. Remember that $P^{*}$ is the solution to the system (4), the upper right derivative of $L_{2}$ can be estimated: 


$$
\begin{aligned}
D^{+} L_{2}= & \operatorname{sgn}\left(S-S^{*}\right)\left(S^{\prime}-\left(S^{*}\right)^{\prime}\right)+\operatorname{sgn}\left(E-E^{*}\right)\left(E^{\prime}-\left(E^{*}\right)^{\prime}\right) \\
& +\operatorname{sgn}\left(I-I^{*}\right)\left(I^{\prime}-\left(I^{*}\right)^{\prime}\right) \\
= & \operatorname{sgn}\left(S-S^{*}\right)\left[A-\frac{k S^{h} I}{S^{h}+\alpha I^{h}}-\mu S-\left(A-\frac{k S^{* h} I^{*}}{S^{* h}+\alpha I^{* h}}-\mu S^{*}\right)\right] \\
& +\operatorname{sgn}\left(E-E^{*}\right)\left[\frac{k S^{h} I}{S^{h}+\alpha I^{h}}-(\mu+q) E-\left(\frac{k S^{* h} I^{*}}{S^{* h}+\alpha I^{* h}}-(\mu+q) E^{*}\right)\right] \\
& +\operatorname{sgn}\left(I-I^{*}\right)\left[q E-(\mu+\delta+\lambda) I-\left(q E^{*}-(\mu+\delta+\lambda) I^{*}\right)\right] .
\end{aligned}
$$

and we all know that when $x>0, \operatorname{sgn}(x)=1 ; x=0, \operatorname{sgn}(x)=0 ; x<0$, $\operatorname{sgn}(x)=-1$.

In (27), there are 8 kinds of situation for the size of $S$ and $S^{*}, E$ and $E^{*}$, $I$ and $I^{*}$, it is enough to analyze the situation of $S>S^{*}, E>E^{*}, I>I^{*}$, while for the other situations, the discussion is similar.

Firstly there is

$$
\begin{aligned}
D^{+} L_{2} & \leq-\mu\left|S-S^{*}\right|-\mu\left|E-E^{*}\right|-(\mu+\delta+\lambda)\left|I-I^{*}\right| \\
& \leq-\mu\left|S-S^{*}\right|-\mu\left|E-E^{*}\right|-\mu\left|I-I^{*}\right| \\
& \leq-\mu L_{2} .
\end{aligned}
$$

Integrate from $t_{0}$ to $t$ on both sides of (28), we have

$$
L_{2}(t)+\mu \int_{t_{0}}^{t} L_{2}(x) \mathrm{d} x \leq L_{2}\left(t_{0}\right)<+\infty .
$$

Since the front set $\Gamma$ has a boundary, $S, E$ and $I$ must have boundaries, and their derivatives are bounded. It means that $L_{2}$ is uniformly continuous.

By Barbalat Lemma in [20], there is

$$
\lim _{t \rightarrow+\infty} L_{2}(t)=0 \text {, }
$$

and the unique endemic equilibrium point $P^{*}$ of model (4) is globally asymptotically stable. This completes the proof.

\section{Brief Summary}

In this paper, we consider the SEIR epidemic model which is different from the classical nonlinear incident rate. We assume that the infectious force is a function of a number ratio of the infective to that of the susceptible which takes the form $\frac{k S^{h} I}{S^{h}+\alpha I^{h}}$, and the regeneration matrix is used to obtain the basic reproductive number $R_{0}$; the existence of equilibrium is obtained by direct calculation.

By constructing the proper Lyapunov functions, we prove that if $R_{0} \leq 1$, there exists only the disease-free equilibrium point which is globally asymptotically stable, and if $R_{0}>1$, there is a unique endemic equilibrium point and this endemic equilibrium point is globally asymptotically stable. 
In the future work, we can further consider adding pulse condition to the model studied in this paper.

\section{References}

[1] Ma, Z.E., Zhou, Y.C. and Wang, W.D. (2004) Mathematical Modeling and Research on the Dynamics of Infectiuos Diseases. Science Press, Beijing.

[2] Kermack, W.O. and McKendrick, A.G. (1927) Cotributions to the Mathematical Theory of Epidemics-I. Proceedings of the Royal Society, 115A, 700-721.

https://doi.org/10.1098/rspa.1927.0118

[3] Cai, Y.L., Kang, Y. and Wang, W.M. (2017) A Stochastic SIRS Epidemic Model with Nonlinear Incidence Rate. Applied Mathematics and Computation, 305, 221-240. https://doi.org/10.1016/j.amc.2017.02.003

[4] Ma, Z.E. and Zhou, Y.C. (2009) Modeling and Dynamics of Infectiuos Diseases. Higher Education Press, Beijing. https://doi.org/10.1142/7223

[5] Zhao, Y. (2013) Analysis of a Dynamic Model of Host-Parasite Interaction with Delay and Treatment. Chin Quart J of Math, 28, 118-128.

[6] Wang, Z.W. and Wang, W.D. (2010) Mathematical Analysis of Immune Response of HIV-I Including Delay. Chin Quarrt J of Math, 25, 45-51.

[7] Zhang, X.Q. and Zhao, Z. (2010) Differential Susceptibility SIR Epidemic Model with Time Delay and Pulse Vaccination. Chin Quart Jof Math, 25, 228-235.

[8] Zhang, J. and Ma, Z.E. (2003) Global Dynamics of an SEIR Epidemic Model with Saturating Contact Rate. Mathematical Biosciences, 185, 15-32. https://doi.org/10.1016/S0025-5564(03)00087-7

[9] Korobeinikov, A. and Maini, P.K. (2004) A Lyapunov Function and Global Properties for SIR and SEIR Epidemiological Models with Nonlinear Incidence. Mathematical Bioscience and Engineering, 1, 57-60. https://doi.org/10.3934/mbe.2004.1.57

[10] Liu, W.M., Hethcote, H.W. and Levin, S.A. (1987) Dynamical Behavior of Epidemiological Models with Nonlinear Incidence Rates. Mathematical Biosciences, 25, 359-380.

[11] Yuan, S.L. and Li, B. (2009) Global Dynamics of an Epidemic Model with a Ratio-Dependent Nonlinear Incidence Rate. Discrete Dynamics in Nature and Society, 609306, 13.

[12] Bain, C. (1990) Applied Mathematical Ecology. Journal of Epidemiology and Community Health, 44, 254. https://doi.org/10.1136/jech.44.3.254-b

[13] Korobeinikov, A. and Maini, P.K. (2005) Nonlinear Incidence and Stability of Infectious Disease Models. Mathematical Medicine and Biology, 22, 113-128. https://doi.org/10.1093/imammb/dqi001

[14] Fred, B. and Carlos, C.C. (2012) Mathematical Models in Population Biology and Epidemiology. 2nd Edition, Springer, Berlin.

[15] Hethcote, H.W. (2000) The Mathematica of Infectious Diseases. SIAM Review, 42, 599-653. https://doi.org/10.1137/S0036144500371907

[16] Capasso, V. and Serio, G. (1978) A Generalizaton of the Kermack-Mckendrick Deterministic Epidemic Model. Mathematical Biosciences, 42, 43-61. https://doi.org/10.1016/0025-5564(78)90006-8

[17] Driessche, P.V.D. and Wathmough, J. (2002) Reproduction Number and Suc-Threshold Endemic Equilibria for Compartmental Models of Disease Transmission. Mathematical Biosciences, 180, 29-48. 
https://doi.org/10.1016/S0025-5564(02)00108-6

[18] Bai, Z.G. (2013) The Basic Reproduction Number of the Periodic Infectious Disease Models. Chinese Journal of Engineering Msthematics.

[19] Elaydy, S. (2004) An Introduction to Difference Equations. Springer, New York, 204-255.

[20] Min, Y.Y. and Liu, Y.G. (2007) Barbalat Lemma and Its Application in System Stability Analysis. Journal of Shandong University, 37, 51-55. 